\title{
Postpartum Depression Without Antenatal Depression in Primiparous Women
}

\author{
Ryuhei Kurashina ${ }^{a}$, Shunji Suzukia, b
}

\section{To the Editor}

Antenatal depression has been suggested to be the strongest predictor of postnatal depression; and in turn postpartum depression has seemed to be the strongest predictor for parenting stress [1-4]. To date, some studies have suggested that maternal depressive symptoms during pregnancy are associated with postpartum depressive symptoms, especially those are depressed during the third trimester of pregnancy [1-4]. In our earlier small study [4], depressive and/or anxiety symptoms showed somewhere during pregnancy in $86 \%$ of the women who had depressive symptoms during postpartum. Therefore, early identification and treatment of perinatal depression has been suggested to be important $[5,6]$. On the other hand, some women develop depressive symptoms during postpartum without any depressive symptoms during pregnancy [4]. Although we have tended to get caught up in the former as "high-risk approach", we must always keep in mind that the latter is present, because it is undeniable that they also have a potential risk of maltreatment.

Based on these backgrounds, we examined the factors associated with the symptoms of maternal depression developing for the first time during postpartum by increasing the number of subjects.

The protocol for this study was approved by the Ethics Committee of Japanese Red Cross Katsushika Maternity Hospital. Informed consent concerning retrospective analyses was obtained from all subjects.

Between April 2016 and March 2018, we requested all Japanese nulliparous women $(\mathrm{n}=1,131)$ who delivered single baby at term at our institute to answer the Whooley's two questions $[7,8]$, a self-administered questionnaire at first, second and third trimesters of pregnancy $(8-11,20-24$ and 34 - 37 weeks of gestation) and postpartum (1 month after delivery). In this study, 29 women were excluded because of

Manuscript submitted October 22, 2021, accepted October 30, 2021

Published online November 20, 2021

aDepartment of Obstetrics and Gynecology, Japanese Red Cross Katsushika Maternity Hospital, Tokyo, Japan

${ }^{b}$ Corresponding Author: Shunji Suzuki, Department of Obstetrics and Gynecology, Japanese Red Cross Katsushika Maternity Hospital, 5-11-12 Tateishi, Katsushika-ku, Tokyo, Japan. Email: czg83542@mopera.ne.jp

doi: https://doi.org/10.14740/jocmr4624 current mental disorders with psychotherapies and/or medications, and 1,097 women of the rest (/1,102: 99.5\%) gave us analyzable answers. The Whooley's two questions have been reported to be used as a screening tool for adult depression including women at perinatal period $[7,8]$. It consists of the following two questions: 1) Have you often been bothered by feeling down, depressed or hopeless during the past 1 month? 2) Have you often been bothered by having little interest or pleasure in doing things during the past month? If at least one of the two questions is "yes", the woman was rated as having depressive symptom.

Using medical charts of the women who responded validly, we examined maternal age, history of mental disorders, delivery mode and the most troubled problem at 2 - 4 weeks after delivery such as feeling of insufficient breastfeeding, baby crying at night, wound pain, breast trouble, et al. "Crying at night" means that baby suddenly starts crying in midnight and does not stop crying for no reason.

Table 1 shows the results of Whooley's two questions during pregnancy and postpartum. The prevalence of women with postpartum depressive symptoms was $13.9 \%(153 / 1,097)$.

Of the 153 women who had depressive symptoms during postpartum, 128 women $(83.7 \%)$ showed depressive symptoms somewhere during their pregnancies, while 25 women (16.3\%) showed no depressive symptoms during pregnancy. As a result of examining their medical charts, seven of the 25 women $(28.0 \%)$ were worried about baby crying at night as shown in Table 2.

Based on the current results, antenatal depressive symptoms seemed to be an obvious predicting factor for postnatal depressive symptoms. The tendency was similar to that in our previous small study [4], and it will support some previous studies [1-3].

On the other hand, $16 \%$ of women who had postpartum depressive symptoms did not have depressive symptoms during pregnancy.

The most common cause of their depressive symptoms was baby crying at night. Pregnancy/delivery is a life-altering event, and everybody is supposed to be aware of that raising baby is not a piece of cake. However, night time waking and crying have been frequently culturally constructed as behavioural sleep "problems" $[9,10]$. Mothers are usually the principal caregivers, providing extensive body contact day and night and prolonged breastfeeding with immediate nurturant response to crying. However, separate sleeping arrangements and delayed response to crying have been recommended reg- 
Table 1. Results of Whooley's Two Questions in 1,097 Primiparous Women During Pregnancy and Postpartum

\begin{tabular}{lllll} 
& First trimester & Second trimester & Third trimester & Postpartum \\
\hline Total & 1,097 & 1,097 & 1,097 & 1,097 \\
"Yes" at least one question & 218 & 44 & 61 & 153 \\
$(\%)$ & 19.9 & 4.0 & 5.7 & 13.9 \\
\hline
\end{tabular}

Data are presented as number and percentage.

ularly, because education related to sleep enhancement has seemed to have no effect on baby crying time [9-11]. To date, there has been some evidence of a strong relationship between sleep disturbance and postpartum depression mainly in intermediate and above mothers who are considered low risk [12]. In addition, an association between poor sleep quality/quantity and symptoms of anxiety, depression and psychosis has been observed [13]. The current result may support these previous studies. Therefore, a useful way to protect the maternal mental health should be searched, rather than disregarding the baby crying at night as a small trouble.

The second most common problem in the women with postpartum depressive symptoms only was breast troubles. There was no significant difference in the prevalence of the two problems by Fisher's exact test $(\mathrm{P}=0.74)$. Breastfeeding is not always easy and the difficulties of breastfeeding are common. Most women have been seemed to experience some breastfeeding discomfort and one or two problems along the way such as sore nipples, breast engorgement and clogged milk ducts. These troubles are common in the early postpartum period and have been reported to be associated with early cessation of breastfeeding and comorbidities such as depression, anxiety, and mastitis [14]. However, early, effective postnatal treatment of the maternal health and breastfeeding problems has been suggested to reduce the risk for poor mental health $[14,15]$. Therefore, these should not be ignored as one of the minor troubles.

The other factors should be also considered in similar ways. Because, postpartum unexplained sadness, anxiety and/ or loneliness of the mothers, once labeled, "baby blues" have been observed to be related to the mothers whose vaginal delivery was not spontaneous and/or large episiotomies were car-

Table 2. Most Troubled Problem in 25 Primiparous Women With Postpartum Depressive Symptoms Without Antenatal Depressive Symptoms at 2 - 4 Weeks After Delivery

\begin{tabular}{lll}
\hline & Number & $\mathbf{\%}$ \\
\hline Baby crying at night & 7 & 28 \\
Breast trouble & 5 & 20 \\
Wound pain, breast trouble & 4 & 16 \\
Insufficient breastfeeding & 4 & 16 \\
Dissatisfaction with partner & 2 & 8 \\
Newborn disease & 2 & 8 \\
For no reason & 1 & 4 \\
Total & 25 & 100 \\
\hline
\end{tabular}

Data are presented as number and percentage. ried out [16].

In the current study, we understand there are some serious limitations such as the small sample size. In this study, we did not examine the women without postpartum depressive symptom as the control group. A large case-control study may be needed to clarify the postpartum problems more.

In conclusion, the prevalence of women with postpartum depressive symptoms was $13.9 \%$. Of them, $16.3 \%$ showed no such symptoms during pregnancy. The most common problem of them was baby crying at night. A useful way to protect the maternal mental health should be searched, rather than disregarding "baby crying at night" as a small trouble.

\section{Acknowledgments}

None to declare.

\section{Financial Disclosure}

This research did not receive any specific grant from funding agencies in the public, commercial, or not-for-profit sectors.

\section{Conflict of Interest}

None to declare.

\section{Informed Consent}

Informed consent concerning retrospective analyses was obtained from all subjects.

\section{Author Contributions}

RK and SS conceived and designed the study, acquired, analyzed and interpreted the data, drafted the paper. SS critically reviewed the manuscript. RK and SS read and approved the final manuscript.

\section{Data Availability}

The authors declare that data supporting the findings of this study are available within the article. 


\section{References}

1. Matthey S, Molgora S. Depression in pregnancy "strongly predicts" depression postpartum: Are we inadvertently misleading clinicians and researchers? J Affect Disord. 2021;295:50-55.

2. Leigh B, Milgrom J. Risk factors for antenatal depression, postnatal depression and parenting stress. BMC Psychiatry. 2008;8:24.

3. Okano T. Pregnancy, delivery and clinical psychiatry practice (in Japanese). Jpn J Psych Treat. 2013;28:545551

4. Suzuki S, Eto M. Screening for depressive and anxiety symptoms during pregnancy and postpartum at a Japanese perinatal center. J Clin Med Res. 2017;9(6):512-515.

5. Royal College of Psychiatrists. Mental health in pregnancy. http://www.rcpsych.ac.uk/healthadvice/problemsdisorders/mentalhealthinpregnancy.aspx. September 9, 2021.

6. Suzuki S, Eto M. Current status of social problems during pregnancy at a perinatal center in Japan. JMA J. 2020;3(4):307-312.

7. Whooley MA, Avins AL, Miranda J, Browner WS. Casefinding instruments for depression. Two questions are as good as many. J Gen Intern Med. 1997;12(7):439-445.

8. The National Institute for Health and Care Excellence. Antenatal and postnatal mental health: clinical management and service guidance. Clinical guideline [CG192]. https://www.nice.org.uk/guidance/cg192. September 9, 2021.

9. Blunden SL, Thompson KR, Dawson D. Behavioural sleep treatments and night time crying in infants: challenging the status quo. Sleep Med Rev. 2011;15(5):327334.

10. Lozoff B, Brittenham G. Infant care: cache or carry. J Pediatr. 1979;95(3):478-483.

11. Bryanton J, Beck CT, Montelpare W. Postnatal parental education for optimizing infant general health and parent-infant relationships. Cochrane Database Syst Rev. 2013;11:CD004068.

12. Bhati S, Richards K. A systematic review of the relationship between postpartum sleep disturbance and postpartum depression. J Obstet Gynecol Neonatal Nurs. 2015;44(3):350-357.

13. Lawson A, Murphy KE, Sloan E, Uleryk E, Dalfen A. The relationship between sleep and postpartum mental disorders: A systematic review. J Affect Disord. 2015;176:6577.

14. Buck ML, Amir LH, Cullinane M, Donath SM, CASTLE Study Team. Nipple pain, damage, and vasospasm in the first 8 weeks postpartum. Breastfeed Med. 2014;9(2):5662.

15. Cooklin AR, Amir LH, Nguyen CD, Buck ML, Cullinane M, Fisher JRW, Donath SM, et al. Physical health, breastfeeding problems and maternal mood in the early postpartum: a prospective cohort study. Arch Womens Ment Health. 2018;21(3):365-374.

16. Thalassinos M, Rouillon F, Engelmann P, Lemperiere T. [Study of the relation of gynecologic and obstetric findings and psychological disorders of pregnancy and the puerperium]. J Gynecol Obstet Biol Reprod (Paris). 1988;17(7):879-887. 